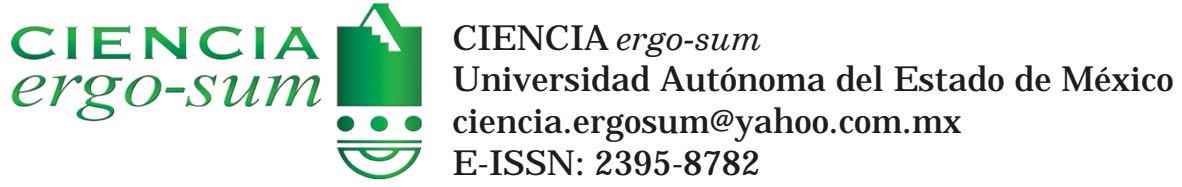

\title{
Eficiencia en el mercado de carne de cerdo en México
}

Rebollar-Rebollar, Samuel; Martínez Damián, Miguel Ángel; Callejas J uárez, Nicolás;

Velázquez-Villalva, Héctor Hugo

Eficiencia en el mercado de carne de cerdo en México

CIENCIA ergo-sum, vol. 26, núm. 3, noviembre 2019-febrero 2020|e65

Universidad Autónoma del Estado de México, México

Esta obra está bajo una Licencia Creative Commons Atribución-NoComercial-SinDerivar 4.0 Internacional.

Rebollar-Rebollar, S., Martínez Damián, M. Á., Callejas J uárez, N. y Velázquez-Villalva, H. H. (2019).

Eficiencia en el mercado de carne de cerdo en México. CIENCIA ergo-sum, 26(3). https://doi.org/10.30878/

ces.v26n3a8 


\title{
Eficiencia en el mercado de carne de cerdo en México
}

Efficiency in the pork market in Mexico

\author{
Samuel Rebollar-Rebollar \\ Universidad Autónoma del Estado de México, México \\ samrere@hotmail.com \\ Miguel Ángel Martinez Damián \\ Colegio de Postgraduados, México \\ angel24559@gmail.com \\ Nicolás Callejas Juárez \\ Universidad Autónoma de Chihuahua, México \\ nicolascallejasjuarez@gmail.com \\ Héctor Hugo Velázquez-Villalva \\ Universidad Autónoma del Estado de México, México \\ hectoreltino@hotmail.com
}

Recepción: 03 de julio de 2017

Aprobación: 21 de junio de 2018

\section{Resumen}

Se valida un modelo de programación no lineal con datos de 2015 para evaluar la eficiencia del mercado de carne de cerdo en México mediante condiciones de optimización de Kuhn-Tucker. Se utilizaron ocho regiones productoras-consumidoras y dos puntos de internación de importaciones: Noroeste, Norte, Noreste, Centro-Oeste, Centro-Este, Sur, Oriente, Península. El modelo subestimó la producción nacional en $0.4 \%$ y sobreestimó el consumo en $2.5 \%$ con un valor social neto de 3789.9 MMDP. La optimización dedujo producción y consumo positivos, así como margen de comercialización superior al costo de transporte, activándose rutas óptimas de distribución. El ajuste entre lo observado y el modelo fue menor a la unidad. Las condiciones de Kuhn-Tucker aplicadas al modelo de optimización permitieron demostrar la eficiencia del mercado porcino.

Palabras ClaVe: carne de cerdo en canal, Kuhn-Tucker, programación no lineal, valor social neto.

\begin{abstract}
To evaluate the efficiency of the pork market in Mexico, a non-linear programming model with 2015 data was validated using Kuhn-Tucker optimization conditions. Eight import-producing regions and two importation points were used: Northwest, North, Northeast, Midwest, Central-East, South, East, and Peninsula. The model underestimated national production by $0.4 \%$, overestimated consumption by 2.5 with a Net Social Value of 3789.9 billion of pesos. The optimization deducted positive production and consumption, marketing margin superior to the cost of transport, activating optimal routes of distribution. Kuhn-Tucker conditions applicate to optimization model let demonstrate the efficiency of the pork market in Mexico.
\end{abstract}

KEYWORDS: pork in carcass, Kuhn-Tucker, nonlinear programming, Net Social Value.

\section{Introducción}

La carne de cerdo es una de las más producidas en el mundo: en 2010 se produjeron 108.9 millones de toneladas (Mt), en 2014 fueron $110.5 \mathrm{Mt}$ y para 2015 se incrementó $0.3 \%$ (110.9 Mt) (USDA, 2016). Para este último año, China fue líder con 51\% (56.6 Mt), la Unión Europea y Estados Unidos aportaron 22.6 y $10.5 \%$ respectivamente (USDA, 2016) y Hong Kong y China sobresalieron con 67.7 y 42.5 kg per cápita (Porcimex, 2017).

En México la producción de carne de cerdo la llevan a cabo 5800 productores. En 2010 el país aportó 1.1 Mt al total mundial (1.2\%) y para $20161.4 \mathrm{Mt}$, lo cual lo posicionó en el lugar décimo sexto. Por entidad federativa, en 2016, Jalisco, Sonora, Puebla, Yucatán, Veracruz, Guanajuato y Michoacán aportaron 0.9 Mt (65.9\%) (Porcimex, 2017).En 
cuanto al consumo, destacaron la Ciudad de México y el Estado de México como los más importantes (Conapo, 2016; SNIIM, 2016; Porcimex, 2017). Sin duda, este volumen obedeció al crecimiento en el número de consumidores, cambios en patrones, gustos y preferencias, así como movimientos en el ingreso per cápita, entre otros (Sosa et al., 2017).

Las condiciones de Kuhn-Tucker, conocidas como Karusch-Kuhn-Tucker (KKT), son requerimientos necesarios y suficientes para que la solución de un problema de programación matemática sea óptima. Se conciben como una generalización del método de los multiplicadores de Lagrange (Andreani et al., 2005; Boyd et al., 2011), utilizados como procedimiento para encontrar máximos y mínimos de funciones de múltiples variables sujetas a restricciones. Este método reduce el problema restringido con $n$ variables a uno $\sin$ restricciones de $n+k$ variables, donde $k$ es igual al número de restricciones, y cuyas ecuaciones pueden ser resueltas por un método convencional.

Estas nuevas variables escalares desconocidas, una para cada restricción, son llamadas multiplicadores de Lagrange (Wallace, 2004). Este método alude a que los puntos donde la función tiene un extremo condicionado a $k$ restricciones está entre los puntos estacionarios de una nueva función sin restricciones construida como una combinación lineal de la función original y las funciones implicadas en las restricciones, cuyos coeficientes son los multiplicadores de Lagrange (Boyd et al., 2011).

Con base en lo anterior, el problema consistió en estimar la función lagrangena ampliada mediante la incorporación del modelo de optimización y sus respectivas restricciones, así como obtener los multiplicadores de Lagrange de la función de aquellos relacionados básicamente con la producción y consumo, precios de mercado tanto al productor como al consumidor y los multiplicadores respectivos que dan constancia de prueba de que el margen de comercialización del producto en cada una de las regiones es superior al costo de distribución, lo cual se convierte en condición necesaria y suficiente para que una ruta de distribución regional del producto se active dentro del modelo.

De este modo, el objetivo del artículo fue optimizar la distribución del mercado de la carne de cerdo en canal en México mediante la validación de un modelo de programación no lineal y las condiciones de optimización de Kuhn-Tucker. La hipótesis central supone que el intercambio entre regiones en producción, consumo e importaciones puede ser mejorado, por lo que se reflejaría en un mayor Valor Social Neto (VSN).

\section{Materiales y métodos}

Con base en Bassols et al. (1992), el país se dividió en ocho regiones productoras, ocho regiones consumidoras (tabla 1) y dos puntos de internación de las importaciones; estos puntos de internación se agregaron a la matriz de regiones productoras, lo que dio un total de 10. Se efectuó el análisis de forma regional debido a que tanto la producción como el consumo de carne de cerdo en México se lleva a cabo en forma distinta y bajo diferentes sistemas productivos; existen regiones en donde el volumen de producción es menor al consumo y requieren que otras con excedentes realicen envío del producto hacia aquellas que son deficitarias.

TABLA 1

Regiones y entidades de México

\begin{tabular}{|ll}
\hline Región & \multicolumn{1}{c}{ Estados } \\
\hline Noroeste (NO) & Baja California, Baja California Sur, Sonora, Sinaloa y Nayarit \\
Norte (NR) & Chihuahua, Coahuila, Durango, San Luis Potosí, Zacatecas \\
Noreste (NE) & Nuevo León y Tamaulipas \\
Centro-Occidente (CO) & Aguascalientes, Colima, Guanajuato, Jalisco, Michoacán \\
Centro-Este (CE) & Ciudad de México, Hidalgo, Estado de México, Morelos, Puebla, Querétaro, Tlaxcala \\
Sur (SU) & Chiapas, Oaxaca y Guerrero \\
Oriente (OR) & Veracruz y Tabasco \\
Península de Yucatán (PE) & Campeche, Yucatán y Quintana Roo \\
\hline
\end{tabular}

Fuente: Bassols et al. (1992). 
El punto de internación 1 (PI1) incorporó las aduanas de Colombia en el estado de Nuevo León, también Nuevo Laredo y Reynosa, pertenecientes al estado de Tamaulipas, y Piedras Negras en el estado de Coahuila, México. De la carne de cerdo importada por México, 90.1\% tuvo como punto de internación PI1, mientras que el resto (9.8\%) tuvo como punto de internación PI2 y se integró por las aduanas de Mexicali y Tijuana, en el estado de Baja California, así como Nogales y San Luis Río Colorado en el estado de Sonora y Ciudad Juárez en el estado de Chihuahua (SIAP, 2016). De esta manera se conformaron diez regiones productoras: ocho nacionales y dos puntos de internación.

Se utilizó un modelo de equilibrio parcial de precios endógenos (modelo espacial) con programación no lineal (programación cuadrática) sin almacenamiento. La función objetivo (función de Valor Social Neto, VSN) describe una situación de optimización que consistió en maximizar el área bajo las curvas de demanda, menos el área bajo las curvas de oferta, menos los costos de comercialización de la carne de cerdo en canal en México sujeta a restricciones de oferta y demanda. El modelo de precios endógenos se aplicó al mercado de la carne de cerdo en canal en México con datos observados en 2015.

El modelo supone la existencia de regiones productoras y consumidoras que comercian un bien homogéneo (Takayama y Judge, 1964), carne porcina en canal, las cuales se encuentran separadas por los costos de transporte, pero no aisladas entre sí.

En la determinación del VSN fue necesario la definición endógena de precios; por lo tanto, se empleó la función inversa de la demanda (Martínez y Hernández, 2012), que para la región $i$ fue

$$
P_{d i}=P_{d i}\left(Y_{d i}\right)=\lambda_{d i}+\omega_{d i} Y_{d i} ; \omega_{d i}<0
$$

Donde:

$P_{d i}=$ precio de demanda de carne de cerdo en canal en la región $i$ en pesos por tonelada

$Y_{d i}=$ cantidad demandada de carne de cerdo en canal en la región $i$ en toneladas

$\lambda_{d i}=$ intercepto de la función de demanda de carne de cerdo en canal en la región $i$

$\omega_{d i}=$ pendiente de la función de demanda de carne de cerdo en canal en la región $i$

De igual forma, para la región $i$ la función inversa de la oferta fue

$$
P_{s i}=P_{s i}\left(X_{s i}\right)=V_{s i}+\eta_{s i} X_{s i} ; \eta_{s i}>0
$$

Donde:

$P_{S i}=$ precio de oferta de carne de cerdo en canal en la región $i$ en pesos por tonelada

$X_{s i}=$ cantidad ofrecida de carne de cerdo en canal en la región $i$ en toneladas

$V_{s i}=$ intercepto de la función de oferta de carne de cerdo en canal en la región $i$

$\eta_{s i}=$ pendiente de la función de oferta de carne de cerdo en canal en la región $i$

El área bajo la curva de demanda $(\mathrm{ABCD})$ se obtuvo al integrar la función inversa de la demanda en el intervalo desde 0 hasta $Y_{d i}^{*}$, donde $Y_{d i}^{*}$, es la cantidad óptima por obtener.

$$
A B C D=\int_{0}^{Y_{d i}^{*}}\left(\lambda_{d i}+\omega_{d i} Y_{d i}\right) d Y_{d i}=\lambda_{d i} Y_{d i}^{*}+\frac{1}{2} \omega_{d i} Y_{d i}^{* 2}
$$

El área bajo la curva de oferta $(\mathrm{ABCO})$, en el intervalo de 0 a $X_{s i}^{*}$, donde $X_{s i}^{*}$ es la cantidad óptima abtener. Se obtuvo como sigue:

$$
A B C O=\int_{0}^{X_{s i}^{*}}\left(V_{s i}+\eta_{s i} X_{s i}\right) d X_{s i}=V_{s i} X_{s i}+\frac{1}{2} \eta_{s i} X_{s i}^{2}
$$


Al incorporar los costos de transporte entre regiones, la función de bienestar social (VSN) para la $n$-región fue

$$
\text { Max. VSN }=\left[\sum_{i=1 \mid}^{8}\left(\lambda_{d i} Y_{d i}+\frac{1}{2} \omega_{d i} Y_{d}^{2}\right)-\sum_{i=1}^{8}\left(V_{s i} X_{s i}+\frac{1}{2} \eta_{s i} X_{s i}^{2}\right)\right]-\sum_{i=1}^{10} \sum_{j=1}^{10} X_{i j} T_{i j}
$$

Donde:

$X_{i j}=$ es la cantidad transportada de la región $i$ a la región $j(\$ / \mathrm{t})$ de carne porcina en canal

$T_{i j}=$ costo de transporte de la región $i$ a la región $j(\$ / \mathrm{t})$ de carne de cerdo en canal $\lambda_{d i}>0$ y $V_{s i}>0$

Otros componentes en el modelo fueron las restricciones de demanda y de oferta. Las primeras requirieron que la suma de la cantidad transportada de carne de cerdo en canal a la región $i$ sea mayor o igual que la cantidad demandada en dicha región. Esto es:

$$
Y_{8} \leq \sum_{j=1}^{10} X_{i j}, \quad \text { para toda } i
$$

Las restricciones de oferta requirieron que la suma de la cantidad transportada de carne de cerdo en canal fuera de la región $i$, sea menor o igual a la producción total de esta carne de cerdo en dicha región:

$$
X_{8} \geq \sum_{j=1}^{10} X_{i j} \text {, para toda } i
$$

Las condiciones de no negatividad implican que $X_{S i} \geq 0, Y_{d i} \geq 0$ y $X_{S} \geq 0$. Se tienen, por tanto, tres variables dentro del modelo: oferta y demanda en cada región (pues una vez que se obtienen, puede generarse el precio a través de la función inversa), y también flujos comerciales entre regiones. Las condiciones de optimización se formularon a través del Lagrangeano.

$$
\begin{aligned}
L=\sum_{i=1}^{8}\left(\lambda_{d i} Y_{d i}\right. & \left.+\frac{1}{2} \omega_{d i} Y_{d i}^{2}\right) \\
& -\sum_{i=1}^{10}\left(V_{s i} X_{s i}+\frac{1}{2} \eta_{s i} X_{s i}^{2}\right) \\
& +\sum_{s=1}^{10} \sum_{d=1}^{8} t_{s d} x_{s d}+\sum_{d=1}^{8} \sigma_{d}\left(\sum_{s=1}^{10} X_{s d}-Y_{d i}\right)+\sum_{s=1}^{8} \sigma_{s}\left(X_{s}-\sum_{d=1}^{8} X_{s d}\right)
\end{aligned}
$$

Para su solución fue necesario imponer condiciones de Kuhn-Tucker (Wallace, 2004; Andreani et al., 2005; Boyd et al., 2011), las cuales se pueden definir y exponer como sigue:

$$
\frac{\partial \mathrm{L}}{\partial \mathrm{Y}_{d i}}=\lambda_{d i}+\omega_{d i} \overline{Y_{d}}-\overline{\sigma_{d}}=0
$$

$\lambda_{d i}+\omega_{d i}\left(\overline{Y_{d}}\right)$ es el precio de mercado al consumidor y $(\overline{\sigma d})$ es el precio óptimo al consumidor.

$$
\left.\frac{\partial L}{X_{s i}}=-\left(V_{s i}+\eta_{s i} X_{s i}\right)+\overline{\sigma_{s}} \leq 0 \mathrm{y}\left(\frac{\partial L}{X_{s i}}\right) \overline{\left(X_{S}\right.}\right)=0, \forall s
$$


$V_{s i}+\eta_{s i} X_{s i}$ es el precio de mercado al productor y $\overline{\sigma_{S}}$ es el precio óptimo al productor.

$$
\begin{gathered}
\frac{\partial L}{\partial X_{s d}}=-t_{s d}+\overline{\sigma_{d}}-\overline{\sigma_{s}} \leq 0 \mathrm{y}\left(\frac{\partial L}{\partial X_{s d}}\right)\left(\overline{X_{s d}}\right)=0, \forall s \mathrm{y} d . \\
\overline{\sigma_{d}}>0, \overline{\sigma_{s}}>0
\end{gathered}
$$

Por lo tanto, cuando el margen de comercialización óptimo $\left(\overline{\sigma_{d}}-\overline{\sigma_{S}}\right)$ es igual al costo de transporte en cada región $\left(-t_{s d}\right)$, se cumple la condición necesaria para que en el modelo óptimo se generen flujos comerciales.

$$
\frac{\partial L}{\partial \sigma_{d}}=\sum_{s=1}^{10} X_{s d}-Y_{d i} \geq 0 \mathrm{y},\left(\frac{\partial L}{\partial \sigma_{d}}\right)\left(\sigma_{d}\right)=0, \forall d
$$

Esta condición (derivada parcial) implica que para que $\sigma_{d} \geq 0$ no tendría que haber exceso de demanda.

$$
\frac{\partial L}{\partial \sigma_{S}}=X_{S}-\sum_{d=1}^{8} X_{S d} \geq 0 \mathrm{y},\left(\frac{\partial L}{\partial \sigma_{S}}\right)\left(\sigma_{S}\right)=0, \forall s
$$

Esta derivada parcial (condición) implica que no debe haber exceso de oferta.

\section{Datos}

La información por entidad federativa sobre producción y exportaciones de carne de cerdo en canal se obtuvo del Servicio de Información Agroalimentaria y Pesquera (SIAP, 2016); después, se restó la exportación a la producción de los estados que reportaron y se sumó la producción de los estados que integran cada una de las regiones para obtener el dato del volumen regional.

$$
S_{i}=Q_{i}-\text { exportaciones }
$$

Asimismo, la información sobre volúmenes importados se obtuvo del SIAP (2016) según claves arancelarias y puntos de acceso al país (aduanas).

El consumo (demanda) de carne porcina en canal de cada una de las ocho regiones del país, se obtuvo como sigue: la población de cada estado (Conapo, 2016) se multiplicó por la disponibilidad per cápita reportado para la zona proveniente de la Confederación de Porcicultores Mexicanos (Porcimex) y fue para la región Norte 5.8 kg, Centro 17.4 kg y Sur 29.5 kg (Porcimex, 2016); después se sumó el consumo de cada uno de los estados que integraron cada región. La Confederación de Porcicultores divide al país en esas tres regiones solamente.

El precio regional de cerdo en canal se obtuvo con el precio de cada entidad que integró la región, el cual se ponderó con la producción (SIAP, 2016). El precio de la carne de cerdo proveniente de EE.UU. hacia los puntos de internación se obtuvo del SNIIM (2016).

El costo de transporte dentro y entre regiones se calculó al multiplicar la distancia (kilómetros) por el costo por kilómetro $(\mathrm{km})$; las distancias $(\mathrm{km})$ provinieron de la Secretaría de Comunicaciones y Trasportes punto a punto (SCT, 2016), además se consideró cada capital de la entidad federativa como punto de referencia y se ponderaron las distancias en cada región. El transporte de canales de cerdo es especializado y tuvo un costo por kilómetro de dos dólares (USD): el equivalente a 35 pesos en el momento de la investigación, el cual se obtuvo a través de entrevista a una empresa que realiza servicios de transporte de carga especializada.

En las ecuaciones de oferta y demanda regionales de carne de cerdo se utilizaron elasticidades precio de la demanda y precio de la oferta regionales inelásticas reportadas por Rebollar et al. (2014), y para los puntos de internación se consideraron las elasticidades nacionales reportadas por Pérez (2010). Las funciones precio-cantidad se calcularon como lo indicaron Alston et al. (1995) y Kawaguchi et al. (1997). 
Se obtuvo el modelo base, conocido como modelo óptimo, y sus resultados tanto a nivel regional como nacional sobre producción, consumo e importaciones y el VSN. Una diferencia porcentual de estimación entre el modelo y los datos observados menor al 10\% se considera como aceptable para realizar el análisis de política. Si tal diferencia resulta negativa/positiva, entonces el modelo subestima o sobreestima los resultados. Una vez estimado y validado el modelo óptimo se procedió a realizar el análisis de las condiciones de Kuhn-Tucker.

Para el modelo base, el precio de la carne de cerdo importada fue $27.4 \$ / \mathrm{kg}$ (obviando proyecciones de incremento en costos de producción internos, producto del aumento en el precio de los insumos por la nueva situación económica de México en 2017, tipo de cambio e incremento en el precio de la gasolina), que se sumó al costo de transporte de los puntos de internación PI1 y PI2.

La información secundaria se procesó y los resultados se obtuvieron con el procedimiento MINOS, contenido en el software GAMS (General Algebraic Modeling System), versión 24.4.2 para Windows 8, Office 2013 y con base en Rosenthal (2008).

\section{Resultados Y Discusión}

El modelo base o modelo óptimo obtenido subestimó a la producción nacional y a su distribución por regiones en $0.4 \%$, esto es, el volumen de producción óptima nacional fue menor al volumen observado en $4655 \mathrm{t}$, cantidad cercana al valor observado en 2015. También, el modelo base sobreestimó al consumo nacional y regional en $0.8 \%$ al pasar de 2100.5 miles t, como el valor observado en 2015 a 2117.7 miles t dadas por el modelo. Adicionalmente, el modelo sobreestimó a la variable importaciones de carne en canal en $2.5 \%$, pues el dato observado fue menor que el del modelo en magnitud equivalente a 21.9 miles t.

Bajo condiciones óptimas y sin distorsiones, una alternativa de política pecuaria en favor del bienestar social sugiere una reducción regional en la producción de carne de cerdo en canal y la maximización del VSN implicaría que en específico las regiones $\mathrm{CO}, \mathrm{CE}$ y la $\mathrm{NO}$ habrían de equilibrar sus volúmenes de producción, equivalente a una reducción de 339, 1020 y 186 t, respectivamente.

En el modelo base se observa un aumento del consumo nacional (producción más importaciones) de carne de cerdo en canal de $0.8 \%$ (17 255 t). En otras palabras, el modelo permite generar una redistribución de estas tres variables del mercado considerada como óptima, una vez que el VSN fue superior al observado en 13.6 MMDP. La disminución en producción nacional de carne en canal de cerdo se resolvería incrementando en aproximadamente 21.9 miles t las importaciones sugeridas por el modelo con el fin de compensar la disminución en la producción. Como el modelo base maximiza el precio de importación y precio doméstico del nivel observado a un nivel óptimo, las regiones que tendrían un incremento mayor en su consumo serían la CO, CE y la NR (cuadro 1).

\section{CUADRO 1}

Producción, consumo e importaciones de carne de cerdo en México bajo condiciones óptimas (2017)

\begin{tabular}{|lrrr}
\hline \multicolumn{1}{c}{ Región } & Datos observados & Modelo base & Diferencia \\
\hline & \multicolumn{2}{c}{ Producción (t) } & \\
Noroeste (NO) & 193739 & 193553 & -186 \\
Norte (NR) & 33393 & 33145 & -248 \\
Noreste (NE) & 31633 & 31426 & -207 \\
Centro-Occidente (CO) & 419748 & 419409 & -339 \\
Centro-Este (CE) & 233277 & 232257 & -1020 \\
Sur (SU) & 76240 & 76192 & -48 \\
Oriente (OR) & 130667 & 128866 & -1801 \\
Península de Yucatán (PE) & 106345 & 105539 & -806 \\
Nacional & 1225042 & 1220387 & -4655 \\
\hline
\end{tabular}


CUADRO 1 (continúa)

Producción, consumo e importaciones de carne de cerdo en México bajo condiciones óptimas (2017)

\begin{tabular}{lrrr}
\hline & \multicolumn{2}{c}{ Importaciones (t) } & \\
Punto de internación 1 & 788977 & 810588 & 21611 \\
Punto de internación 2 & 86497 & 86777 & 280 \\
Nacional & 875474 & 897365 & 21891 \\
& & Consumo (t) & \\
Noroeste (NO) & 80397 & 80423 & 26 \\
Norte (NR) & 124481 & 126296 & 1815 \\
Noreste (NE) & 50139 & 50538 & 399 \\
Centro-Occidente (CO) & 354833 & 363390 & 8557 \\
Centro-Este & 769221 & 774512 & 5291 \\
Sur (SU) & 284737 & 284747 & 10 \\
Oriente (OR) & 303029 & 303891 & 862 \\
Península de Yucatán (PE) & 133680 & 133975 & 295 \\
Nacional & 2100517 & 2117772 & 17255 \\
VSN (MMDP) & 3776.3 & 3789.9 & 13.6 \\
\hline
\end{tabular}

Fuente: elaboración propia de acuerdo con los resultados del modelo base o modelo óptimo. MMDP: miles de millones de pesos.

\section{Consumo óptimo}

Con base en las condiciones de optimización de Kuhn-Tucker, para que existan valores positivos en términos de consumo y producción óptimos de carne porcina en canal en cada región se requiere que en cada una de las regiones el precio de mercado estimado en la ecuación del precio tanto para la demanda como en la oferta sea igual al precio óptimo dado por en el modelo base (cuadro 2).

\section{CUADRO 2}

Obtención de las condiciones de Kuhn-Tucker para la demanda de carne porcina en canal

\begin{tabular}{lrccc}
\hline Región & $\begin{array}{c}\text { Consumo } \\
\text { óptimo (t) }\end{array}$ & $\begin{array}{c}\text { Precio de } \\
\text { mercado (\$)* (A) }\end{array}$ & $\begin{array}{c}\text { Precio } \\
\text { óptimo (\$) (B) }\end{array}$ & $\begin{array}{c}\text { Diferencia } \\
\text { (A-B) }\end{array}$ \\
\hline NO & 80423.2 & 31410.0 & 31410.0 & 0 \\
NR & 126296.1 & 33700.0 & 33700.0 & 0 \\
NE & 50538.0 & 34577.0 & 34577.0 & 0 \\
CO & 363390.0 & 34200.0 & 34200.0 & 0 \\
CE & 774511.7 & 35190.0 & 35190.0 & 0 \\
SU & 284746.7 & 36640.0 & 36640.0 & 0 \\
OR & 303890.7 & 36590.0 & 36590.0 & 0 \\
PE & 133974.5 & 38340.0 & 38340.0 & 0 \\
\hline
\end{tabular}

Fuente: cálculos propios de acuerdo con los resultados del modelo base.

Nota: en la última columna se observan ceros debido a que es la evidencia de cumplimiento de la condición matemática de Kuhn-Tucker donde el precio de mercado y el precio óptimo, por el lado de la demanda, deben ser iguales. El segundo lo da la salida del modelo y el primero se construye con información de datos observados. 
Para la demanda, la función precio estimada para la región noroeste $(\mathrm{NO})$ fue $P_{N O}=5575143-68.932 Y_{N O}$, lo cual significa un consumo óptimo de $80423.217 \mathrm{t}$; un precio de $P_{N O}=5575143-68.932(80423.217)=$ $31410.0 \$ /$ t. Este valor es lo que se considera como el precio de mercado, en tanto que el precio óptimo $\left(\sigma_{N O}\right)$ dado por el modelo para la región NO fue 31410 \$/t; por ello, la diferencia aritmética en ambos precios (el de mercado menos el precio óptimo) fue 0 y con esto se cumple la condición matemática de consumos óptimos positivos. De forma similar para el resto de las regiones del país, los consumos óptimos, precios de mercado y precios óptimos se presentan en el cuadro 2. La región CE consume 37\% y la NE solamente 2\%; el mayor consumo depende del número de consumidores, más que del precio.

\section{Producción óptima}

Para la cantidad ofertada, la ecuación del precio de mercado, conocido como precio endógeno para la misma región (NO), fue $P_{N O}=-5508641+28.605 X_{N O}$ y el volumen de producción óptima fue $193552.553 \mathrm{t}$ de carne porcina; con esto, el precio de mercado fue 27929.778 \$/t, en tanto que el precio óptimo (cuadro 3), dado por el modelo base para la misma región, fue 27929.778 \$/t, por lo que la diferencia en ambos precios es 0 . Esta condición se consideró necesaria y suficiente para que existan precios y consumos óptimos positivos. Para el resto de las regiones, el cálculo fue similar. En este caso, 38\% del consumo nacional tendría como origen el mercado internacional (PI1) y la región CO debería producir 20\% como principal consumidora.

\section{CUADRO 3}

Obtención de las condiciones de Kuhn-Tucker para la oferta de carne porcina en canal

\begin{tabular}{lcccc}
\hline Región & $\begin{array}{c}\text { Producción } \\
\text { óptima (t) }\end{array}$ & $\begin{array}{c}\text { Precio de } \\
\text { mercado (\$/t) (A) }\end{array}$ & $\begin{array}{c}\text { Precio } \\
\text { óptimo (\$/t) (B) }\end{array}$ & $\begin{array}{c}\text { Diferencia } \\
\text { (A-B) }\end{array}$ \\
\hline NO & 193552.553 & 27929.778 & 27929.778 & 0 \\
NR & 33145.411 & 32193.778 & 32193.778 & 0 \\
NE & 31425.980 & 32435.779 & 32435.779 & 0 \\
CO & 419408.814 & 33444.778 & 33444.778 & 0 \\
CE & 232257.209 & 34707.778 & 34707.778 & 0 \\
SU & 76191.570 & 34901.780 & 34901.780 & 0 \\
OR & 128886.025 & 35608.778 & 35608.778 & 0 \\
PE & 105539.055 & 37698.778 & 37698.778 & 0 \\
PI1 & 810587.659 & 31169.778 & 31169.779 & 0 \\
PI2 & 86776.631 & 27924.782 & 27924.782 & 0 \\
\hline
\end{tabular}

Fuente: elaboración propia de acuerdo con los resultados del modelo base.

Nota: en la última columna se observan ceros debido a que es la evidencia de cumplimiento de la condición matemática del modelo lagrangeano donde el precio de mercado y el precio óptimo, por el lado de la oferta, deben ser iguales. El segundo lo da la salida del modelo y el primero se construye con información de datos observados.

\section{Flujos comerciales óptimos}

Con base en los resultados del modelo base o modelo óptimo, la condición para que existan flujos comerciales entre regiones es que el diferencial entre los precios óptimos sea igual o mayor que 0 (cuadro 4). En este caso todas las regiones presentaron un margen mayor que 0 . 


\section{CUADRO 4}

Márgenes de comercialización óptimos de carne porcina en canal

\begin{tabular}{|lccc}
\hline Región & $\begin{array}{c}\text { Precio óptimo } \\
\text { al consumidor } \mathbf{( \$ / t )}(\mathbf{A})\end{array}$ & $\begin{array}{c}\text { Precio óptimo } \\
\text { al productor } \mathbf{( \$ / t )}(\mathbf{B})\end{array}$ & $\begin{array}{c}\text { Margen } \mathbf{( \$ / \mathbf { t } )} \\
(\mathbf{A}-\mathbf{B})\end{array}$ \\
\hline $\mathrm{NO}$ & 31410.0 & 27929.8 & 3480.2 \\
$\mathrm{NR}$ & 33700.0 & 32193.8 & 1506.2 \\
$\mathrm{NE}$ & 32600.0 & 32435.8 & 164.2 \\
$\mathrm{CO}$ & 34200.0 & 33444.8 & 755.2 \\
$\mathrm{CE}$ & 35190.0 & 34707.8 & 482.2 \\
$\mathrm{SU}$ & 36640.0 & 34901.8 & 1738.2 \\
$\mathrm{OR}$ & 36590.0 & 35608.8 & 981.2 \\
$\mathrm{PE}$ & 38340.0 & 37698.8 & 641.2 \\
\hline
\end{tabular}

Fuente: elaboración propia de acuerdo con los resultados del modelo base.

Las rutas de comercialización o distribución de carne de cerdo que se activaron entre regiones (cuadro 5) se debió a que la magnitud del margen de comercialización fue igual o mayor que 0 , fortaleciéndose la condición de optimización de Kuhn-Tucker derivada del modelo lagrangeano. Otras rutas de comercialización de carne porcina en canal entre regiones no se visualizan en dicho cuadro debido a que el margen fue menor, en magnitud, que el costo de transporte.

CUADRO 5

Carne porcina en canal. Rutas de comercialización óptimas por región

\begin{tabular}{|c|c|c|c|}
\hline Ruta activada (Xsd) & Margen (\$/t) & Costo de transporte $(\$ / t)$ & Diferencia (\$) \\
\hline $\mathrm{X}_{1,1}(\mathrm{NO}$ a $\mathrm{NO})$ & 3480.2 & 3480.0 & 0.2 \\
\hline $\mathrm{X}_{1,4}(\mathrm{NO}$ a $\mathrm{CO})$ & 6270.2 & 6268.4 & 1.8 \\
\hline $\mathrm{X}_{2,5}(\mathrm{NR}$ a $\mathrm{CE})$ & 2996.2 & 2992.1 & 4.1 \\
\hline $\mathrm{X}_{3,5}(\mathrm{CO}$ a $\mathrm{CE})$ & 2996.2 & 2821.6 & 174.6 \\
\hline $\mathrm{X}_{3,7}(\mathrm{NE}$ a OR $)$ & 4154.2 & 4153.0 & 1.2 \\
\hline $\mathrm{X}_{4,4}(\mathrm{CO}$ a $\mathrm{CO})$ & 755.2 & 753.2 & 2.0 \\
\hline $\mathrm{X}_{4,5}(\mathrm{CO}$ a $\mathrm{CE})$ & 1745.2 & 1740.8 & 4.4 \\
\hline $\mathrm{X}_{5,6}(\mathrm{CE}$ a $\mathrm{SU})$ & 1932.2 & 1932.0 & 0.2 \\
\hline $\mathrm{X}_{5,7}(\mathrm{CE}$ a OR $)$ & 1882.2 & 1880.0 & 2.2 \\
\hline $\mathrm{X}_{6,6}(\mathrm{SU}$ a $\mathrm{SU})$ & 1738.2 & 1738.2 & 0.0 \\
\hline $\mathrm{X}_{7,7}(\mathrm{OR}$ a OR $)$ & 981.2 & 980.0 & 1.2 \\
\hline $\mathrm{X}_{7,8}(\mathrm{OR}$ a $\mathrm{PE})$ & 2731.2 & 2730.7 & 0.5 \\
\hline $\mathrm{X}_{8,8}(\mathrm{PE}$ a $\mathrm{PE})$ & 641.2 & 640.0 & 1.2 \\
\hline $\mathrm{X}_{9,2}$ (PI1 a NR) & 2530.2 & 2530.0 & 0.2 \\
\hline $\mathrm{X}_{9,3}(\mathrm{PI} 1 \mathrm{a} \mathrm{NE})$ & 1430.2 & 1430.0 & 0.2 \\
\hline $\mathrm{X}_{9,5}(\mathrm{PI} 1$ a CE$)$ & 4020.2 & 4016.4 & 3.9 \\
\hline $\mathrm{X}_{9,7}(\mathrm{PI} 1 \mathrm{a}$ OR $)$ & 5420.2 & 5420.0 & 0.2 \\
\hline $\mathrm{X}_{10,2}(\mathrm{PI} 2 \mathrm{a} \mathrm{NR})$ & 5775.2 & 5774.7 & 0.5 \\
\hline
\end{tabular}

Fuente: elaboración propia de acuerdo con los resultados del modelo base. 


\section{Análisis Prospectivo}

En México indudablemente el mercado de la carne porcina en canal en el mediano plazo es sensible a toda una gama de factores que determinan su dinámica, ya sea que tales factores se ubiquen en el lado de la producción, en importaciones o en el consumo nacional. En general, existe evidencia en donde la producción se ha visto tergiversada por variaciones en precios de los insumos de un periodo a otro (García et al., 2005), en especial los del sorgo grano (Rebollar et al., 2005) y precios de la gasolina con impacto en costos de transporte de animales vivos (Alonso Pesado y Rodríguez de Jesús, 2017) vinculados al costo de producción (Medina, 2012), retiro de subsidios gubernamentales a la producción (García et al., 2005), discontinuidad en apoyos al productor por cada animal sacrificado en rastro TIF o aplicación de aranceles a las importaciones como medida de apoyo a producción nacional (Gómez et al., 2011; Velázquez et al., 2016).

Es prudente hacer notar que el mercado porcino en canal también es sensible al efecto de dos importantes macroprecios: $a$ ) depreciación cambiaria y $b$ ) impuestos a la producción nacional. El primero protege a productores e incrementaría en el mediano y largo plazo la planta productiva, pero desprotege a consumidores nacionales. En el segundo, con demandas y ofertas inelásticas regionales, se desprotege a la producción nacional, importaciones y a consumidores nacionales, el precio al productor disminuye, el precio al consumidor aumenta, el bienestar de la sociedad disminuye, en tanto que el impacto del impuesto lo reciben más los consumidores que productores debido a la inelasticidad de la demanda.

Al simular un escenario, sobre el modelo base, de 20.9\% de depreciación cambiaria ocurrida en 2016 (Alonso y Rodríguez, 2017), los resultados indican que un beneficio (protección) a la producción nacional en $0.4 \%$ en relación con la obtenida en el modelo óptimo es que las regiones productoras como la Centro-Occidente habrían de recibir el impacto mayor, el consumo habría sido menor en $0.6 \%$ y las importaciones tenderían a reducirse en $2 \%$. En términos de regiones consumidoras, aquellas que por lo regular presentan más dinamismo en el consumo recibirían el efecto mayor de la política, como la Centro-Este y Centro-Occidente.

Actualmente, se plantea que mucha carne daña la salud humana, incluso una disponibilidad per cápita de carne de cerdo mayor a $17 \mathrm{~kg}$ por año puede conducir a enfermedades coronarias, por lo que una política impositiva sobre producción se ha estado debatiendo en foros e investigaciones. Sobre esta base, si el gobierno mexicano gravara la oferta de carne porcina con $16 \%$ de impuesto, se esperaría que los efectos económicos en el mercado nacional recaigan en una reducción de producción nacional equivalente a $0.42 \%$, las importaciones podrían reducirse en $1.0 \%$ y el consumo disminuya en $0.7 \%$. En consecuencia, los precios óptimos al consumidor habrán de tener un incremento regional, en relación con el modelo base en 3.9, 6.2 y 7.8\% para las regiones más dinámicas que son la CO, CE y SU debido a que el productor podría transferir la mayor parte del impuesto al consumidor sin que disminuya sustancialmente la cantidad.

\section{Conclusiones}

El modelo permitió obtener el volumen y precio óptimos de mercado para la carne de cerdo en México, así como demostrar la hipótesis de una distribución óptima del producto entre regiones. Bajo un escenario de depreciación cambiaria, los productores nacionales mejoran su bienestar y una política al valor agregado apenas disminuye la producción nacional de la carne de cerdo en canal. De igual modo, el modelo puede utilizarse para el análisis de la aplicación de escenarios de política comercial como aranceles, cuotas, depreciación cambiaria, subsidios o impuestos sobre los cuales se base el interés de dicha política.

\section{REFERENCiAS}


Alonso Pesado, F. A. y Rodríguez de Jesús, E. (2017). El aumento de los precios de los combustibles automotrices y su efecto en el subsector pecuario. Los porcicultores y su entorno, 19(115), 42-48.

Andreani, R., Martinez, J. M., \& Schuverdt, M. L. (2005). Technical note: On the relation between constant positive linear dependence condition and quasinormality constraint qualification. Journal of optimization theory and applications, 125(2), 473-485.

Alston, J. M., Norton, G. W., \& Pardey, P. G. (1995). Science under scarcity: Principles and practice for agricultural research evaluation and priority setting. Ithaca, New York: Cornell University Press.

Bassols, A. B., Delgadillo, M. J. y Torres, T. F. (Eds.) (1992). El desarrollo regional de México: teoría y práctica. Libros de la Revista Problemas del Desarrollo. UNAM: México.

Boyd, S., Parikh, N., Chu, E., Peleato B., \& Eckstein, B. (2011). Distributed optimization and statistical learning via the alternating direction method of multipliers. Retrevied from https://www.nowpublishers.com/ article/Details/MAL-016.

Conapo (2016). Consejo Nacional de Población. Disponible en https://www.gob.mx/conapo.

García, S. J. A., Rebollar, R. S. y Rodríguez, L. G. (2005). Integración vertical y competitividad del sector porcino en México. Comercio Exterior, 55(6), 524-532.

Gómez, T. G., Rebollar, R. S., Hernández, M. J., \& Guzmán, S. E. (2011). Effect of the tariffs in the competitiveness of the mexican pork industry. Tropical and Subtropical Agroecosystems, 14(2), 537-542.

Kawaguchi, T., Susuki, N., \& Kaiser, M. (1997). A spatial equilibrium model for imperfectly competitive milk markets. American Journal of Agricultural Economics, 79, 851-859.

Martínez, D. M. A. y Hernández, O. J. (2012). Importaciones de granos básicos y precio interno en México: un enfoque de sistema de demanda inverso. Agricultura, Sociedad y Desarrollo, 9, 401-410.

Medina, R. S. (2012). México en el mercado internacional de cerdo. Comercio Exterior, 63(6), 7-10.

Pérez, V. F. C., García, M. R., Martínez, D. M. A., Mora-Flores, J. S., Vaquera, H. H. y González, E. A. (2010). Efecto de las importaciones de la carne de porcino en el mercado mexicano, 1961-2007. Revista Mexicana de Ciencias Pecuarias, 1(2), 115-126.

Porcimex (Confederación de Porcicultores Mexicanos). (2017). Estadisticas. Disponible en http://www.porcimex. org/estadisticas/analiticos/mcarne.htm.

Rebollar, R. S., García, S. J. A. y Rodríguez, L. G. (2005). La política cambiaria y el mercado del sorgo en México. Comercio Exterior, 55(5), 394-401.

Rebollar, R. A., Gómez, T. G., Hernández, M. J., Rebollar, R. S. y González, R. F. J. (2014). Comportamiento de la oferta y demanda regional de carne de cerdo en canal en México, 1994-2012. Revista Mexicana de Ciencias Pecuarias, 5(4), 377-392.

Rosenthal, E. R. (2008). GAMS. A User's Guide. Washington, D. C.: GAMS Development Corporation.

SCT (Secretaría de Comunicaciones y Transportes). (2016). Rutas punto a punto. Disponible en http://www. sct.gob.mx/rutaspuntoapunto.

SNIIM (Sistema Nacional de Información e Integración de Mercados) (2016). Secretaría de Economía. Disponible en http://www.economia-sniim.gob.mx/nuevo/mapa.asp.

SIAP (Servicio de Información Agroalimentaria y Pesquera). (2016). Estadísticas. Disponible en http://www. siap.gob.mx/optestadisticasiacon2016parcialsiacon-zip/

Sosa, U. M. E., Martínez, C. F. E., Espinosa, G. J. A. y Buendía, R. G. (2017). Contribución del sector pecuario a la economía mexicana. Un análisis desde la Matriz Insumo Producto. Revista Mexicana de Ciencias Pe- 
cuarias, $8(1), 31-41$.

Takayama, T., \& Judge, G. G. (1964). Spatial equilibrium and quadratic programming. Journal of Farm Economics, 46(1), 67-93.

USDA (United States Department of Agriculture). (2016). Disponible en http://www.usda.gov/wps/portal/ usda/usdahome.

Velázquez, V. H. H., Gómez, T. G., Rebollar, R. S. y Martínez, C. F. E. (2016). Efectos regionales y nacionales sobre la producción y consumo de carne de cerdo con la aplicación de aranceles a carne importada, en $\mathrm{V}$. B. A. Cavalloti., V. B. Ramírez. y V. J. A. Cesín V. J. A. (Eds.), Ganadería, Sociedad y Recursos Naturales (pp. 215-228). Chapingo: Universidad Autónoma Chapingo.

Wallace, B. (2004). Constrained optimization: Kuhn-Tucker conditions. Retrevied from http://www.worldcolleges.info/sites/default/files/enggnotes/kuhn-tuker.pdf.

\section{BY-NC-ND}

